\title{
The Evolving Role of 18F-FDG PET/CT in Assessment of Primary and Secondary Peritoneal Malignancies
}

\author{
Yakout $\mathrm{MS}^{1}$, Eweis $\mathrm{AFM}^{2}$ and Elnaggar $\mathrm{AA}^{3}$ \\ ${ }^{1}$ Professor of Radiology, Al-Azhar University, Egypt \\ ${ }^{2}$ Faculty of Medicine, Cairo University, Egypt \\ ${ }^{3}$ Assisstant Professor of Radiology, Al-Azhar University, Egypt
}

Received date: April 04, 2018; Published date: April 27, 2018

*Corresponding author: Dr. Ahmed Fathy Mohamed Eweis, Mohamed Salem street, Manial, Cairo, Egypt, Tel: +201001361136; Email: drfathy83@gmail.com

\begin{abstract}
Introduction: The peritoneum is the serous membranous lining of the abdominal cavity and organs. A diverse group of malignancies are known to involve the peritoneum. Peritoneal malignancy may either be primary or secondary; the latter is also referred to as peritoneal carcinomatosis. Secondary peritoneal malignancies are the commonest group of peritoneal malignancies. Imaging plays a vital role in the evaluation of patients with suspected or proven peritoneal malignancy. The combination of imaging both tumor function and anatomy has clear advantages in oncological imaging; that's why 18F-FDG- PET/CT has improved the sensitivity and specificity of detection of various neoplastic diseases.
\end{abstract}

Material and Methods: This is a retrospective study carried out from October 2015 to February 2018 for patients who have done PET/ CT scans, whether for pathologically proven primary tumor evaluation, staging refinement or therapy response assessment, or patients with suspected peritoneal malignancy depending on clinical, laboratory or conventional imaging findings. A total number of 50 patients were included in our study (20 males and 30 females)

Results and Conclusion: Evaluation of peritoneal malignancies with 18F-FDG PET/CT is rewarding and our study demonstrates that 18F-FDG PET/CT adds substantially to CT scanning alone in the detection of peritoneal malignancies. It is a useful diagnostic tool especially when peritoneal biopsy is either unavailable or inappropriate.

Keywords: PET/CT; CT; PET; Peritoneum; Mesothelioma; Carcinomatosis; Omental caking; Peritoneal carcinomatosis

\section{Introduction}

The peritoneum is the largest serous membrane in the body and the one with the most complex structure. The omentum is a double-layered extension of the peritoneum that connects the stomach to adjacent organs. The peritoneal reflections form the greater and lesser omenta [1]. Primary peritoneal tumors are much less common than secondary peritoneal tumors. The most common primary peritoneal tumor is mesothelioma. Malignant peritoneal mesothelioma is a rare tumor with a variable appearance at CT [1]. Secondary peritoneal malignances are by far much more common than primary peritoneal tumors. The peritoneum is a preferred site of metastasis of several primary malignancies, principally ovarian cancer in women and colorectal carcinoma in men, as well as primary gastric, pancreatic, and hepatic carcinoma. Distant metastases to the peritoneum from breast and lung carcinoma are also not uncommon [2].

\section{Material and Methods}

This is a retrospective study carried out from October 2015 to February 2018. for patients who have done PET/CT scans, whether for pathologically proven primary tumor evaluation, staging refinement or therapy response assessment, or patients with suspected peritoneal malignancy depending on clinical, laboratory or conventional imaging findings. A total number of 50 patients were included in our study (20 males and 30 females, their age ranged between 16-86 years (mean age 55.06 years)).

\section{Reference standard}

The reference standard to determine the accuracy of the imaging findings included histopathology and follow-up studies for the assessment of the primary tumor and the peritoneal metastatic lesions. 


\section{Imaging protocol}

Integrated PET/CT scanner (GE Discovery VCT 64) is utilized. This dedicated system integrates a PET scanner with a multisection helical CT scanner and permits the acquisition of co-registered CT and PET images in one session.

\section{Patient preparation}

A full history was obtained from all patients \& past history of therapy regimens. All patients were asked to fast for six hours prior to the scan. All metallic items were removed from the patient, including, pants with zipper, bra, belts, bracelets, etc. and the patients were given a gown to wear. An I.V. cannula was inserted in the patient's antecubital fossa (or any other applicable peripheral vein) for administration of 18F-FDG and iodinated contrast material. The patients were instructed to avoid strenuous activity prior to the examination and following injection of the radioisotope to avoid physiologic muscle uptake of FDG. Serum glucose was routinely measured prior to $18 \mathrm{~F}$ FDG injection, and fasting levels were 70-150ng/dl. In case of diabetic patients should not have regular insulin administered subcutaneously within four hours of having FDG administered. Recent serum creatinine level should be available before injection of intravascular iodinated contrast material in all patients. In patients with impaired renal functions iodinated contrast material is avoided.

\section{Dosage administration}

We administered 10-20mCi (370 MBq; approximate dose to patient, $3-5 \mathrm{MBq} / \mathrm{Kg}$ ) of $18 \mathrm{~F}-\mathrm{FDG}$ 45-60 minutes before examination.

\section{Examination time}

We performed topogram images for guidance, low dose non enhanced CT scan first, then a whole body PET study followed by diagnostic (+/- enhanced) whole body CT scan. The whole study took approximately 20-30 minutes.

\section{PET technique}

PET was performed following the non enhanced CT study without moving the patient. Approximately six to seven bed positions are planned in the three dimensional acquisition mode with 3-5 minute acquisition at each bed position.

\section{Diagnostic CT technique}

The contrast enhanced helical CT was performed following injection of $80 \mathrm{~mL}$ of a low-osmolarity iodinated contrast medium at a rate of $4 \mathrm{ml} / \mathrm{sec}$ by using a power injector. For a typical whole body PET -CT study (neck, chest, abdomen, and pelvis), scanning began at the level of the skull base and extended caudally to the level of the upper thighs. Typical scanning parameters would be a collimator width of $5.0 \mathrm{~mm}$, pitch of 1.5 , gantry rotation time of 0.8 second, and field of view of $50 \mathrm{~cm}$. The helical data are retrospectively reconstructed at $1 \mathrm{~mm}$ intervals.

\section{PET/CT fusion}

Hundreds of trans-axial PET and CT images were first reconstructed. These are then reformatted into coronal and sagittal images to facilitate image interpretation. For each of these sets of PET \& CT images, corresponding "fusion" images were generated.

For semi quantitative assessment, regions of interest (ROIs) were put over FDG avid lesions on the attenuation- corrected PET or PET/CT fusion images. The standardized uptake value (SUV) was automatically calculated by the workstation software as the ratio between the accumulation of $18 \mathrm{~F}-\mathrm{FDG}(\mathrm{MBq} / \mathrm{ml})$ in an area of interest (drawn on the images corrected for attenuation) and the activity administered (MBq) for the weight ( $\mathrm{kg}$ ) according to the following formula:

SUV = ROI activity $(\mathrm{MBq} / \mathrm{ml}$ (/injected dose $/ \mathrm{MBq} /$ body weight )kg

\section{Statistical analysis}

Results are expressed as mean \pm standard deviation or number (\%). Statistical Package for Social Sciences (SPSS) was used for data analysis. P value $\leq 0.05$ was considered significant.

Data were coded as $(+1)$ for group A and $(-1)$ for group B (where group A includes patients considered positive by CT alone and group B by combined CT \& PET) then entered in SPSS. Data was summarized using mean, standard deviation (SD), standard error of the mean (SE). Comparison was done using one-way analysis of variance (ANOVA) test. The calculated $\mathrm{P}$ value was calculated at $\alpha$ level of 0.05 .

\section{Results}

Our study included 50 patients, 20 males and 30 females. Age of patients ranged from 16 to 86 years with mean age of 55.06 years. Male to female ratio was 2:3; presenting as following:

1) 47 cases referred for PET/CT scan with pathologically proven primary tumor whether for staging refinement, therapy response assessment or regular follow-up.

2) 3 cases referred for PET/CT scan for occult primary tumor detection; diagnosed with conventional radiological examination / clinical suspicious as metastases of unknown primary.

Patients diagnosed with primary peritoneal malignancies were 3 patients; whereas metastatic peritoneal malignancies were diagnosed in 47 patients.

The malignancies showing metastatic peritoneal involvement in our study in decreasing order of frequency, were ovarian cancer (19 patients), colorectal cancer (10 patients), pleural mesothelioma, and pancreatic cancer (3 patients each) , breast cancer, uterine cancer, prostate cancer, and transitional cell urinary bladder cancer (2 patients each), lung cancer, 


\section{Current Trends in Clinical \& Medical Imaging}

appendicular mucinous carcinoma, Non-Hodgkin Lymphoma and hepatocellular carcinoma (1 patient each). Whereas primary peritoneal malignances were primary peritoneal mesothelioma

(2 patients) and primary peritoneal carcinoma (1 patient) (Figure 1)

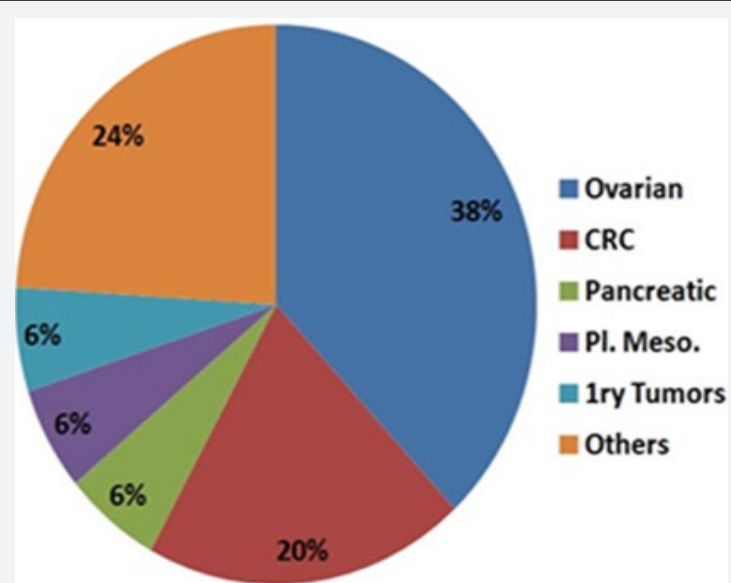

Figure 1: Graph showing prevalence of malignances involvement.

Table 1: Overview of patterns of malignant peritoneal involvement.

\begin{tabular}{|c|c|}
\hline Malignancy & Patterns of Peritoneal Involvement \\
\hline Primary peritoneal mesothelioma & Omental caking with large peritoneal masses formation \\
\hline Primary peritoneal carcinoma & Nodular / mass formation \\
\hline Metastatic ovarian cancer & Omental caking, mixed and nodular patterns \\
\hline Metastatic colon cancer & Nodular and mixed patterns \\
\hline Metastatic uterine cancer & Nodular and mixed patterns \\
\hline Metastatic hepatocellular carcinoma & Nodular pattern \\
\hline Metastatic pancreatic cancer & Nodular pattern \\
\hline Metastatic breast cancer & Nodular pattern \\
\hline Metastatic pleural mesothelioma & Mixed pattern \\
\hline Metastatic prostate cancer & Nodular pattern \\
\hline Metastatic lung cancer & Nodular pattern \\
\hline Metastatic UB cancer & Nodular pattern \\
\hline Non-Hodgkin's lymphoma & Smudged and nodular patterns \\
\hline
\end{tabular}




\section{Current Trends in Clinical \& Medical Imaging}

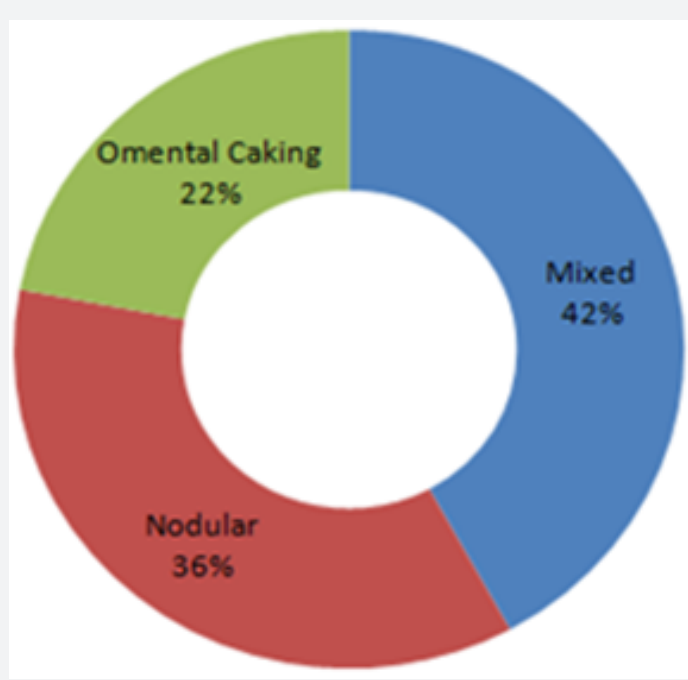

Figure 2: Graph showing commonest patterns of peritoneal involvement.

The most common pattern of peritoneal involvement was mixed in 21 patients (42\%), nodular deposits in 18patients (36\%) and omental caking in 11 patients (22\%) (Figure 2 \& Table 1). The common sites of peritoneal involvement were pelvic reflections (45\%), greater omentum (35\%), and small bowel mesentery (4\%).

The most common malignancy showing peritoneal involvement was ovarian cancer (Fig. 3)seen in 38\% of patients (19 patients) having omental caking as the commonest pattern in $47.36 \%$ ( 9 patients) of these patients followed by mixed peritoneal disease in $31.57 \%$ ( 6 patients) and nodular pattern in $21.05 \%$ ( 4 patients). The predominant sites of peritoneal involvement in ovarian cancer were pelvis followed by greater omentum and small bowel mesentery (Figure $3 \& 4$ ).

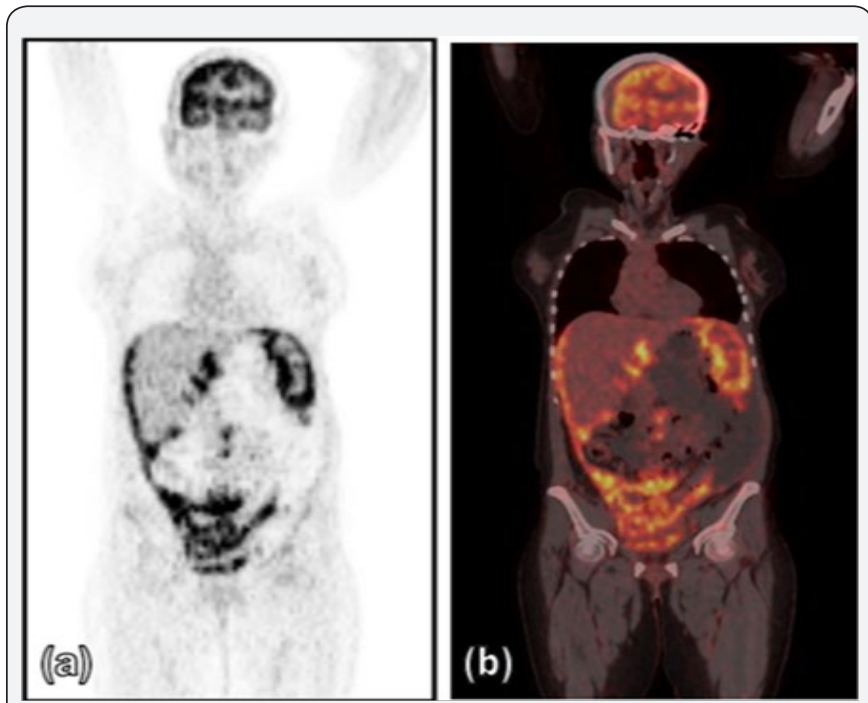

Figure 3: Diffuse metastatic peritoneal carcinomatosis in a known patient with ovarian cancer. (a \& b) PET and fused PET/ CT images showing diffuse avid FDG uptake in pelvic cavity as well as peritoneal reflections wrapping liver and spleen eliciting SUVmax up to 10

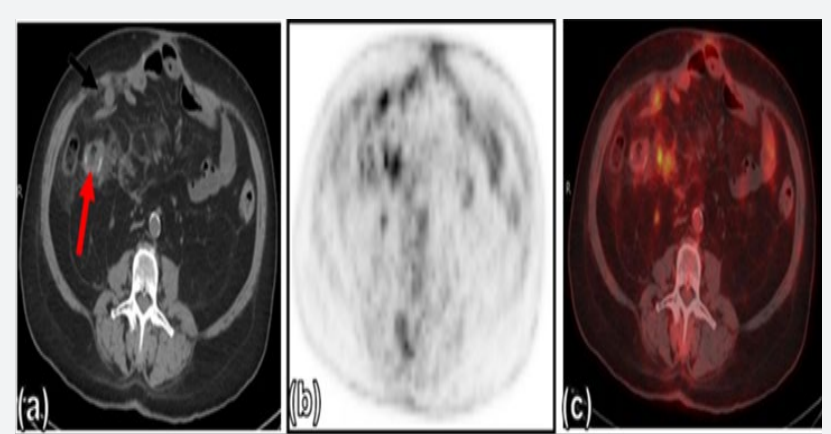

Figure 4: Anastomotic site recurrence and peritoneal deposit in a known patient of adenocarcinoma of the colon after right hemicolectomy. ( $a, b$ \& $c$ ) axial non-contrast CT, PET \& fused PET/CT images demonstrating FDG avid anastomotic site wall thickening (red arrow) as well as discrete omental soft tissue nodule (black arrow) eliciting SUVmax of 5.5 \& 4 respectively.

The second most common malignancy with peritoneal carcinomatosis was colorectal cancer (Figure 4). Perirectal and pericolonic mesentery were the commonest site of involvement in these cases.



Figure 5: hepatic capsular deposits in a known patient of colon cancer. ( $a, b$ \& c) axial non-contrast CT, PET \& fused PET/CT images demonstrating focal FDG avid uptake at hepatic capsule (arrows) with no corresponding masses in CT, eliciting SUVmax of 4.5 .

Predominant pattern in pancreatic, breast, prostate, lung and urinary bladder cancers was nodular pattern. Nodular or 


\section{Current Trends in Clinical \& Medical Imaging}

curvilinear increased FDG uptake along the liver surface was a characteristic FDG PET/CT finding seen in five cases of metastatic peritoneal malignances in our study with no corresponding finding on CT images (Figure 5).

Regarding FDG avidity by the peritoneal malignancies; our study revealed SUVmax ranging from 3 up to 15. The highest FDG uptake was seen in primary peritoneal mesothelioma (Figure 6)

Ascites is a frequent associated finding with different peritoneal malignances in our study; whether primary or secondary. Regarding primary peritoneal mesothelioma ascites was present in all cases ( 2 cases). Whereas in metastatic peritoneal carcinomatosis ascites was present in 33 cases out of 47 cases $(70 \%)$. Ascites was present in 15 out of 19 cases with metastatic ovarian cancer $(80 \%$ of metastatic ovarian cancer cases). Other metastatic peritoneal malignances associated with ascites were colon cancer, uterine cancer, HCC, NHL and pleural mesothelioma (18 cases). FDG avidity was demonstrated in 10 cases out of 35 cases where ascites were present giving SUVmax ranging from 2.5 up to 4 .

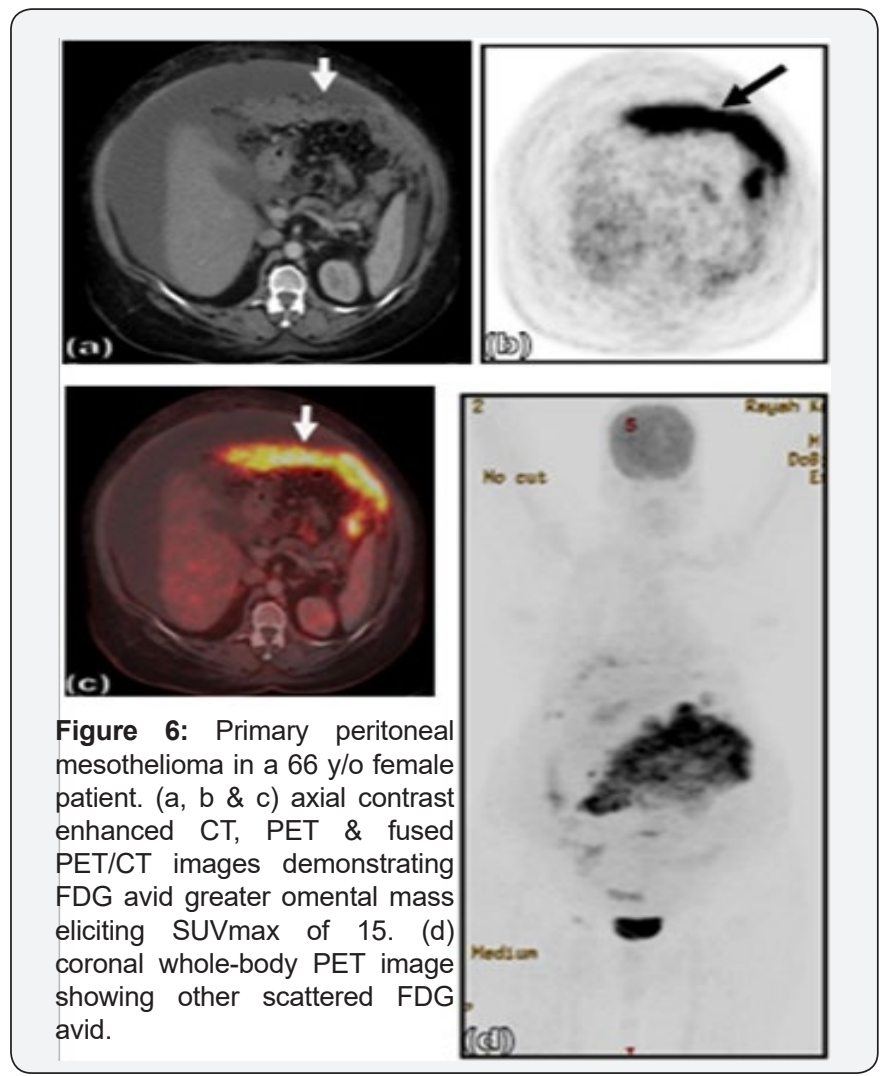

Around $50 \%$ of patients had enlarged abdominal and pelvic lymph nodes associated with metastatic peritoneal malignancies. CT was positive for peritoneal disease in 45 of the 50 patients in our study; however when PET was evaluated together with CT, there were 50 out of 50 positive cases giving p value of 0.02167 indicating significance statistical difference (Table 2).

Table 2: Summary statistics of group A and B (where group A includes patients considered positive by $\mathrm{CT}$ alone and group $\mathrm{B}$ by combined CT \& PET).

\begin{tabular}{|c|c|c|c|c|}
\hline Group & N & Mean & SD & SE \\
\hline A & 50 & 0.8 & 0.60609 & 0.08571 \\
\hline B & 50 & 1 & 0 & 0 \\
\hline
\end{tabular}

\section{Discussion}

The peritoneum is the largest serous membrane in the body and the one with the most complex structure. The omentum is a double-layered extension of the peritoneum that connects the stomach to adjacent organs. The peritoneal reflections form the greater and lesser omenta, and the natural flow of peritoneal fluid determines the route of spread of peritoneal malignancies [1].

Primary peritoneal tumors are much less common than secondary peritoneal tumors. Primary peritoneal tumors can be classified histologically based on the cell lineage of origin into

a) Mesothelial cell origin (mesothelioma)

b) Epithelial cell origin (peritoneal serous neoplasm)

c) Smooth muscle origin and

d) Tumors of uncertain origin (desmoplastic round cell tumor or solitary fibrous tumor) [2].

The most common primary peritoneal tumor is malignant peritoneal mesothelioma which is a rare tumor with a variable appearance at CT. It is commonly associated with ascites, irregular or nodular peritoneal thickening and omental involvement ranging from finely infiltrated fat with a "smudged" appearance to discrete omental nodules or omental caking and sometimes manifests as a large mass in the upper abdomen with minimal ascites and discrete nodules scattered over the peritoneum [1]. Patients with malignant peritoneal mesothelioma may present with mild vague symptoms including abdominal pain, distention, anorexia and weight loss. Because of these vague symptoms, the disease is generally already advanced at presentation [4]. Malignant primary peritoneal mesothelioma may be indistinguishable from carcinomatosis when the predominant imaging findings are multifocal peritoneal nodules and omental involvement. Additional findings to support the diagnosis of peritoneal malignant mesothelioma include the lack of evidence of a primary malignancy or metastasis elsewhere and the lack of lymphadenopathy within the abdomen [5]. Secondary peritoneal malignances are by far much more common that primary peritoneal tumors. The peritoneum is a preferred site of metastasis of several primary malignancies, principally ovarian cancer in women and colorectal carcinoma in men, as well as primary gastric, pancreatic, and hepatic carcinoma. Distant metastases to the peritoneum from breast and lung carcinoma are also not uncommon [5].

The CT appearances of peritoneal metastasis are as follows:

a) Smudged peritoneal fat

b) Discrete solid or cystic nodules / masses, 
c) Multi-focal masses and coalescent peritoneal / omental nodules (omental caking) [1].

Good knowledge of routes by which peritoneal malignancies spread and the dynamics / flow directions of peritoneal circulation is very important in recognizing and detecting such malignances. There are four routes of peritoneal malignances spread

1) Direct invasion

2) Intraperitoneal seeding

3) Lymphatic extension and

4) Haematogenous spread [2].

Ovarian cancers are the most common primary tumors with peritoneal metastasis because they are primarily epithelial cancers; hence, tumor cells are easily washed off the ovary into the peritoneal circulation leading to seeding at several sites. Further, the peritoneal fluid moves upwards, because of pressure gradients in the abdominal cavity. FDG PET/CT for ovarian cancers is indicated for restaging after recurrence [6].

Several FDG PET/CT patterns of peritoneal carcinomatosis have been reported, although not specific to malignancy, and no reliable relationships between patterns and primary cancer types have been described. Findings may include single or multiple enhancing glucose hypermetabolic peritoneal nodules. Peritoneal nodules may become confluent, forming omental cake. When diffuse tumor spread occurs, nodular thickening of the peritoneum with contrast enhancement and increased metabolic activity is seen [7].

Nodular or curvilinear uptake along the liver surface is a characteristic FDG PET finding and had no corresponding finding on CT images. Spread of deposits to the right subhepatic and subphrenic spaces is frequently seen, especially in ovarian cancer [8].

Ascites is a common coexisting finding with peritoneal malignances. There are two main mechanisms causing ascites

1) The main cause is the subphrenic lymphatic vessels becoming obstructed by carcinomatosis, meaning they are unable to carry out their usual function of draining peritoneal fluid.

2) Excess production of peritoneal fluid, resulting from an increase in capillary permeability, which is caused by the tumor cells secreting vascular permeability factor, with protein and albumin accumulating in the abdominal cavity [2].

Due to increased metabolic activity in malignant tissue, FDG PET/CT can identify peritoneal malignancies by increased tracer uptake and the correlation of the tracer distribution with the anatomic structures depicted by CT; together enhance the diagnostic outcome. Patients with peritoneal malignancies may demonstrate ascites, peritoneal thickening, seeding nodules and omental caking [9].

Another benefit of PET/CT is the coverage of the whole body providing information about the presence of any other distant metastases, for example lung or skeletal metastases which change the patient treatment plan [9]. The presence of peritoneal metastasis alters tumor staging, with implications regarding primary treatment and patient outcome. Peritoneal involvement is one of the most significant prognostic indicators in ovarian and colorectal carcinoma, more powerful than the extent of local spread or lymph node involvement. Early diagnosis of peritoneal metastasis is important because low-volume disease may be suitable for cytoreduction (debulking) surgery and hyperthermic intraperitoneal chemotherapy (HIPEC) [10].

\section{Conclusion}

Evaluation of peritoneal malignancies with 18F-FDG PET/ CT is rewarding and our study demonstrates that 18F-FDG PET/ CT add substantially to CT scanning alone in the detection of peritoneal malignancies. It is a useful diagnostic tool especially when peritoneal biopsy is either unavailable or inappropriate.

\section{References}

1. Yoo E, Kim JH, Kim MJ, Yu JS, Chung JJ, et al. (2007) Greater and Lesser Omenta: Normal Anatomy and Pathologic Processes. Radiographics 27(3): 707-720.

2. Diop AD, Fontarensky M, Montoriol PF, Da Ines D (2014) CT imaging of peritoneal carcinomatosis and its mimics. Diagn Interv Imaging 95(9): 861-872.

3. Wasnik AP, Maturen KE, Kaza RK, Al-Hawary MM, Francis IR (2015) Primary and secondary disease of the peritoneum and mesentery: review of anatomy and imaging features. Abdom Imaging 40(3): 626642.

4. Heller DR, Chiuzan C, Taub RN, Leinwand JC, Greene AM, et al. (2017) Recurrence of Optimally Treated Malignant Peritoneal Mesothelioma with Cytoreduction and Heated Intraperitoneal Chemotherapy. Ann Surg Oncol 24(13): 3818-3824.

5. Silva J, Magalhães MJ, Duarte H, Fernandes C, Alves SR, et al. (2013) CT and PET-CT findings of peritoneal carcinomatosis. ECR 2013/ C-2345.

6. Puranik AD, Purandare NC, Agrawal A, Shah S, Rangarajan V (2014) Imaging spectrum of peritoneal carcinomatosis on FDG PET/CT. Jpn J Radiol 32(10): 571-578.

7. Anthony MP, Khong PL, Zhang J (2009) Spectrum of (18)F-FDG PET/ CT appearances in peritoneal disease. AJR Am JRoentgenol 193(6): W523-W529.

8. Suzuki A, Kawano T, Takahashi N, Lee J, Nakagami Y, et al. (2004) Value of 18F-FDG PET in the detection of peritoneal carcinomatosis. Eur J Nucl Med Mol Imaging 31(10): 1413-1420.

9. Klumpp BD, Schwenzer N, Aschoff P, Miller S, Kramer U, et al. (2013) Preoperative assessment of peritoneal carcinomatosis: intraindividual comparison of 18F-FDG PET/CT and MRI. Abdom Imaging 38(1): 6471.

10. Turlakow A, Yeung HW, Salmon AS, Macapinlac HA, Larson SM (2003) Peritoneal carcinomatosis: role of (18)F-FDG PET. J Nucl Med 44(9): 1407-1412. 

(C) Commons Attribution 4.0 License DOI: 10.19080/CTCMI.2018.02.555790
Your next submission with Juniper Publishers will reach you the below assets

- Quality Editorial service

- Swift Peer Review

- Reprints availability

- E-prints Service

- Manuscript Podcast for convenient understanding

- Global attainment for your research

- Manuscript accessibility in different formats ( Pdf, E-pub, Full Text, Audio)

- Unceasing customer service

Track the below URL for one-step submission https://juniperpublishers.com/online-submission.php 